\title{
De las primeras iniciativas de medicina laboral en México: el hospital de mineros de Real del Monte
}

\author{
José Luis Gómez y Carlos Agustín Rodríguez-Paz ${ }^{2}$ \\ ${ }^{1}$ El Colegio de Michoacán, Michoacán; '2Instituto Mexicano del Seguro Social, Hospital General de Zona 50, Servicio de Cirugía General, San Luis \\ Potosí. México
}

\begin{abstract}
Resumen
A pesar de la legislación de Otto von Bismarck (1815-1898) sobre derechos de seguridad social formulada en 1883 en Alemania donde se señala que es deber del Estado promover el bienestar de todos los miembros de la sociedad, particularmente de los más débiles y necesitados utilizando los medios con los que dispone, y las propuestas de leyes contra accidentes expedidas el 30 de abril de 1904 en el Estado de México, en el México del porfiriato no se consideró brindar atención médica formal a los trabajadores, salvo algunas empresas ferrocarrileras, no se construyeron hospitales para atender a pacientes con enfermedades laborales. Una de estas excepciones fue el Hospital del Mineral de Real del Monte de Pachuca, fundado a finales del siglo XIX y después de que la Compañía minera pasó a los norteamericanos en 1906, se acordó que la empresa adquiriera el hospital y lo equipara con los adelantos médicos y quirúrgicos de la época para la inmediata atención de lesiones, en especial de tipo ortopédico, lo que permitió curar heridas y la rehabilitación. Este nosocomio constituye uno de los antecedentes más antiguos en México en las tres disciplinas: ortopedia, medicina del trabajo y rehabilitación. Dejó de funcionar en 1982 y actualmente es un museo con un rico acervo documental y de instrumental de las disciplinas mencionadas.
\end{abstract}

PALABRAS CLAVE: Historia de la medicina. Hospitales. Ortopedia. Rehabilitación. Cirugía.

\begin{abstract}
Despite the legislation of Otto von Bismarck (1815-1898) on social security rights formulated in 1883 in Germany where it is stated that it is the duty of the State to promote the welfare of all members of society, particularly the weakest and most needy, using the means available to them, and the proposals of laws against accidents issued on April 30, 1904 in the State of Mexico in 1904, in the Mexico of the Porfirio Díaz era, providing workers with formal medical care was not contemplated, except in the case of some railway companies, hospitals for the care of patients with occupational diseases were not built. One of these exceptions was the Hospital del Mineral del Real del Monte de Pachuca, founded in the late nineteenth century and after the mining company passed to the Americans in 1906, it was agreed that the company acquired the hospital and equated it with the medical and surgical advances of the time for immediate care of injuries, especially of the orthopedic type, which enabled not only the healing of wounds, but also rehabilitation. This hospital is one of the oldest in Mexico with regard to three disciplines: orthopedics, occupational medicine and rehabilitation. It ceased to operate in 1982, and currently it is a museum with a rich collection of documents and instruments related to the aforementioned disciplines.
\end{abstract}

KEY WORDS: History of medicine. Hospitals. Orthopedics. Rehabilitation. Surgery.

Correspondencia:

José Luis Gómez

E-mail: amoyot!@hotmail.com
Fecha de recepción: 19-02-2016

Fecha de aceptación: 17-03-2016

DOI://dx.doi.org/10.24875/GMM.18002438
Gac Med Mex. 2018;154:263-266

Disponible en PubMed www.gacetamedicademexico.com 


\section{Introducción}

A partir del texto de Bernardino Ramazzini (Morbis artificum diatriba o Acerca de la salud de los trabajadores) de 1700 , se estableció una correlación entre las enfermedades que aparecen como consecuencia de las actividades laborales y sus diversas divisiones (masajistas, mineros, químicos, alfareros, etcétera). ${ }^{1}$

A finales del siglo XIX se identificó la necesidad de atender a los trabajadores de las fábricas y la industria en la legislación formulada por Bismarck, con lo que se inició la seguridad social. ${ }^{2}$ Durante la Primera Guerra Mundial se estableció como principio impedir que los trabajadores de las fábricas dejaran de laborar, ya que se observó que era un factor decisivo en las victorias (producir armamento para acabar con el enemigo). 3,4 En Latinoamérica, alrededor de los años veinte del siglo $X X$ se iniciaron movimientos populares en pro de atender las demandas de salud de los trabajadores.

El artículo 27 de la Constitución de México fue un icono mundial de legislación sobre seguridad laboral, al obligar a los patrones a mejorar las condiciones de salud de los trabajadores y permitir que fueran atendidos de manera oportuna. ${ }^{5}$ En México, desde el siglo XVII se establecieron medidas en las Leyes de los Reinos de Indias, ${ }^{6}$ por las cuales los patrones de minas e ingenios azucareros estaban obligados a mantener un hospital al lado de las instalaciones. Hace una década, el licenciado Felipe Remolina Roqueñí recabó información sobre el derecho laboral en nuestro país. ${ }^{7}$

Fue en 1948 cuando surgió una corriente médica formal, encabezada por el doctor Jorge Fernández Osorio, pionero de la medicina laboral en México, quien fue nombrado médico de puesto de fábrica del Instituto Mexicano del Seguro Social. ${ }^{5}$ En 1968, como parte de un acuerdo entre la Universidad Nacional Autónoma de México y el Instituto Mexicano del Seguro Social se iniciaron los cursos de la especialidad de Medicina del Trabajo. ${ }^{8}$

Antes de 1948 existen interesantes antecedentes de fábricas o negocios que impulsaron medidas para evitar que sus trabajadores enfermaran o dejaran de laborar por los inconvenientes del clima o los riesgos de trabajo propios de sus actividades. Uno de estos ejemplos fue Ferrocarriles de México, que en 1888 ya tenía un sistema de atención médica tanto para pasajeros como para los trabajadores, caracterizado por una red donde había puestos de socorro en las estaciones intermedias a los puntos de los largos trayectos y que más tarde en la Revolución de 1910 a 1921 sirvieron para dar atención a los soldados en combate..$^{9,10}$

\section{El concepto laboral en el porfiriato}

En palabras de Guadalupe Nava Oteo, México surgió como una potencia minera, especialmente como productor de plata; al conocerse en el mundo dicha riqueza, despertó el interés de los inversionistas extranjeros, cuya penetración en esta actividad se intensificó durante el porfiriato. ${ }^{11,12}$ Ingleses y norteamericanos fueron los principales inversionistas de capital en los grandes yacimientos de Zacatecas y Guanajuato, ${ }^{13}$ así como en pequeñas ciudades mineras como Real del Monte en Pachuca, Hidalgo ${ }^{14}$ Con la introducción de capital extranjero se dieron transformaciones profundas en los procesos productivos debido a la enorme cantidad de mineral extraído y la tecnología utilizada. Sin embargo, la llegada de nuevos dueños a las compañías mineras (ingleses y americanos) no cambió las condiciones laborales.

Los barreteros (como se conocía a los mineros por el uso de la barrena) cumplían una jornada laboral de 12 horas, eran contratados sin prestaciones, sus salarios eran de los más bajos, estaban sujetos a condiciones de trabajo insalubres, sufrían malos tratos por parte de los capataces y enfrentaban discriminación ante empleados extranjeros. Los trabajadores que se oponían al progreso y a la paz impuesto por el general Porfirio Díaz de inmediato eran reprimidos.

El 1 de junio de 1906, trabajadores mexicanos de la empresa minera Cananea Consolidated Copper Company decidieron protestar contra los malos tratos y malas condiciones de trabajo en que se encontraban y convocaron a todos los mineros del país a una huelga general con el objetivo de obtener jornadas de trabajo más justas, un salario más equitativo al de sus compañeros mineros norteamericanos 0 europeos (pidieron un incremento de 45 a 50 centavos por hora), así como la modernización y el mejoramiento de las condiciones de trabajo. ${ }^{15}$ Buscaban poner fin a un problema que ocurría en la mayoría de las minas: los accidentes de trabajo, que mermaban la calidad de vida de los empleados sin que estos recibieran remuneración alguna. Como dato adicional, durante 1910 ocurrieron 63 accidentes en Real del Monte y tres años después, en 1913, 98 accidentes. ${ }^{16}$ Para 
1916, año en que la Legislación Social presentó a Venustiano Carranza el Proyecto de Ley de Accidentes, habían ocurrido $104 .^{17}$

A la huelga de Cananea seguiría la de Río Blanco (huelga en una fábrica de tejidos de Río Blanco, Veracruz, el 7 de enero de 1907) y otras rebeliones que prepararon el camino para iniciar lo que sería después la revolución social en México y la creación de la fracción del artículo 123 constitucional en la que se establecía un seguro de incapacidad auspiciado por los patrones con compañías o empresas particulares que tendrían la responsabilidad de pagar los riesgos de trabajo según cantidades valuada (1931). ${ }^{17}$

\section{La inversión minera en el porfiriato}

Sin duda, la minería ha sido de enorme importancia en la historia de México. La abundancia de minerales en nuestro país siempre ha sido una de sus mayores riquezas. La explotación de los metales preciosos ha estado presente en la historia, desde los alejandrinos en Egipto, los alquimistas de la Edad Media y los Estados modernos a partir del siglo XV, que impulsaron y fomentaron la búsqueda y explotación de minas de oro y plata para llenar sus arcas. Con el hallazgo de un nuevo continente (América), se descubrieron nuevas fuentes de abastecimiento, lo que permitió la conquista y colonización de las nuevas tierras.

La minería constituyó, junto con la agricultura y el comercio, el fundamento de la estructura económica colonial. Durante los primeros 10 años del siglo XIX, la producción de plata llegó a niveles nunca alcanzados. Los años de lucha por la independencia provocarían una fuerte crisis en la minería. ${ }^{18}$

Como se mencionó, la minería se vio favorecida durante el porfiriato por la llegada de inversionistas extranjeros, principalmente de origen inglés y americano, que incorporaron elementos tecnológicos que les permitió obtener mayor cantidad de plata. Ejemplo de ello fue la compra de la Sociedad Aviadora de Minas de Real del Monte y Pachuca por parte de la United States Smelting Refining and Mining Company, en una operación de \$255 400 pesos. ${ }^{19}$ Bajo esta administración, la empresa vivió un gran auge; aun cuando inició sus labores años antes de la Revolución Mexicana (1910), esta no le afectó y al llegar la década de 1930, el distrito explotado por la empresa americana se convirtió en el primer productor mundial de plata. Al final de la Segunda Guerra Mundial, la caída del precio de la plata y los impuestos que se aplicaron durante la presidencia del licenciado Miguel Alemán Valdés provocaron que los inversionistas norteamericanos abandonaran las minas, ante lo cual el gobierno mexicano decidió comprar las acciones en $3.5 \mathrm{mi}-$ llones de dólares para impedir el cierre de tan importante fuente de trabajo en la región, convirtiendo a la compañía de Real del Monte en la primera empresa paraestatal.

\section{El hospital de Real del Monte de Pachuca}

La modernidad prometía grandes ganancias, pero la política monetaria del gobierno de Porfirio Díaz de implantar el patrón oro, las bajas cotizaciones del precio de la plata y la exigencia de una fuerte inversión para adoptar la nueva tecnología dieron como resultado que la Sociedad Aviadora de Minas de Real del Monte y Pachuca fuera adquirida el 12 de febrero de 1906 por United States Smelting Refining and Mining Company, que de inmediato introdujo adelantos tecnológicos, como fue el uso de la electricidad.

Entre septiembre y diciembre de 1906, la empresa norteamericana decidió adquirir el hospital minero de Real del Monte y establecer un servicio médico moderno y bien aprovisionado para conseguir que los enfermos fueran oportuna y esmeradamente atendidos. ${ }^{20}$ Para solventar parte de los gastos, la empresa aplicó a los empleados y operarios de las minas un descuento de 2 a $4 \%$, según requiriera el caso, así como una cuota especial de 5 centavos por cada individuo para la construcción del hospital. ${ }^{21}$ Así, barreteros y empleados de compañías mineras como la Blanca, Santa Ana, San Rafael, entre otras, contribuyeron con un porcentaje de su sueldo a la edificación del hospital.

El resultado fue la rehabilitación y refundación del Hospital Minero de la Compañía de Real del Monte y Pachuca en 1907, cuyo personal contaba con un cirujano en jefe, dos cirujanos ayudantes, una enfermera en jefe, cinco enfermeras y un boticario. ${ }^{21}$ Ubicada en el sur del Estado de Hidalgo y muy diferente a los planteamientos porfiristas, la compañía invirtió en la salud de sus trabajadores y construyó una unidad médico-quirúrgica integrada por dos grandes salas, cada una equipada con 12 camas, con espacio adicional para otras 12, para una capacidad total de 48 camas. ${ }^{21}$ La botica quedó instalada y equipada en noviembre de 1907, donde el responsable mezclaba plantas con algunos productos químicos para preparar las recetas indicadas por el médico. ${ }^{22}$ El equipo de 
radiología fue un ejemplo de la avanzada tecnología con la que contaba el hospital: estaba provisto de ruedas, con lo que era posible desplazarlo a las salas del hospital para tomar placas a los pacientes sin necesidad de trasladarlos a la sala de rayos $X$.

El hospital disponía de una sala de curaciones, donde se recibía a los mineros que habían sufrido algún accidente. Un área que ocupó la atención del director del hospital, el doctor A. J. Hoskins, fue la de cirugía, equipada con una mesa de operaciones transparente (que permitía la toma de placas en plena cirugía), pinzas para biopsia, agujas y suturas de diferentes calibres, ampolletas de éter y cloroformo y tanques de oxígeno, entre otros implementos quirúrgicos.

El hospital también contaba con una sala de rehabilitación, donde actualmente se preserva un acervo de férulas de inicios del siglo XX, único en el país, en el que se puede observar tractores de ortejos con los que se mantenían estirados los dedos fracturados de manos o pies para que consolidaran los huesos, férulas cribadas de alambrón o acero inoxidable que eran empleadas para fijar lesiones de tobillo, tibia y peroné, así como férulas de columna cervical y lumbar, lo que deja entrever las principales lesiones que se atendían.

Llama la atención que el hospital dispusiera de una rueda marina para reforzar los músculos de hombro, brazo y antebrazo, escalones con barandales y poleas para recobrar el movimiento de la parte afectada, indicios de una incipiente medicina de rehabilitación en México. También contaba como instrumentos de cirugía ortopédica, entre los que destaca el equipo portátil diseñado por el doctor Albin Lambotte, cánulas Jackson, endoscopios y cistoscopios, ${ }^{22}$ así como una silla de rueda y una camilla para trasladar a los pacientes al hospital.

El hospital dejó de brindar servicios médicos en 1982, cuando los trabajadores del hospital fueron incorporados a los servicios de salud del Instituto Mexicano del Seguro Social. Quedó abandonado hasta octubre de 2004, cuando se rescató el inmueble y se convirtió en el Centro Cultural Nicolás Zavala, fundando el Museo de Medicina Laboral.

\section{Agradecimientos}

Por su apoyo, a la licenciada Belem Oviedo Gámez, directora del acervo del Archivo Histórico de Real del Monte, así como a los licenciados Aracely Monroy Pérez, Cosmelia Ortiz Velázquez y Héctor Alejandro Ruiz Sánchez.

\section{Bibliografía}

1. López-Piñero JM. Medicina, historia y sociedad. Antología de clásicos médicos. Tercera edición. España: Ariel; 1973. p. 164-165.

2. Dimitrijevic M, Obradovic G. Funding social insurance. Facta Universitatis (Law and Politics). 2005;3(1):53-61.

3. Gomero-Cuadra R, Zevallos-Enríquez C, Llapyesan C. Medicina del trabajo, medicina ocupacional y del medio ambiente y salud ocupacional. Rev Med Hered. 2006;17(2):105-108.

4. Schott H. Crónica de la medicina. Tercera edición. México: Intersistemas; 2003. p. 390.

5. Nava HR. Antecedentes históricos de la salud en el trabajo. En: Barquín-Calderón M. Sociomedicina. Cuarta edición. México: Méndez Oteo; 1994: p. 533-536.

6. Título XX, de los mineros y azogueros. En: Recopilación de las Leyes de los Reinos de Indias mandadas a imprimir y publicar por la Majestad Católica del Rey don Carlos II. Madrid: Universidad de Antioquía; 1791.

7. Remolina-Roqueñí F. El derecho burocrático en México. México: Secretaría de Gobernación; 2006.

8. Facultad de Medicina. Plan único de especialidades médicas: Medicina del Trabajo. México: Universidad Nacional Autónoma de México; 2003. Disponible en: http://edumed.imss.gob.mx/cst/Acrobat/PUEM\%20MT.pdf.

9. Fajardo-Ortiz G. Los espacios médico-hospitalarios para los ferroviarios (1850-1981). Rev Fac Med UNAM. 2000;43(3):107-109.

10. Rodríguez-Paz CA, Vázquez-Ortega R. Los trenes hospital de la Revolución Mexicana. Cir Gral. 2009;31(1):46-50.

11. Nava-Oteo G. La minería bajo el porfiriato. En: México en el siglo XIX (1821-1910). México: Nueva Imagen; 1980. p. 339-379.

12. von Mentz B. Trabajo minero y control social durante el porfiriato. Las operaciones de dos poblaciones contrastantes. Historia Mexicana. 2001;50(3):555-607.

13. Meyer-Cosío FJ. La minería en Guanajuato. México: El Colegio de Michoacán/Universidad de Guanajuato; 1998. p. 65-98.

14. Flores-Clair E. Minería y población, Real del Monte 1791-1865. Dimensión Antropológica. 1997;11:7-35. Disponible en: http://www.dimensionantropologica.inah.gob. $m x / ? p=1388$.

15. Cárdenas-García N. La huelga de Cananea en 1906. Una interpretación. Estudios Sociológicos. 1998;16(46):117-146.

16. Licona-Duarte VM. Los mineros "cornish" en el distrito minero de Pachuca y Real del Monte: una minería étnica en México (1849-1906). México: Universidad Nacional Autónoma de México; 1998. p. 5.

17. Benejam MA, Brown-Villalba C, Celis L, Flores-Hernández B, et al. Historia del Instituto Mexicano del Seguro Social. Los primeros años 1943-1944. México: Instituto Mexicano del Seguro Social; 1980, p. 19.

18. Urrutia M, Nava-Oteo G. La minería (1821-1880). En: Cardoso C. México en el siglo XIX (1821-1910). México: Nueva Imagen; 1992. p. 119

19. Ortega-Morel J. Tecnología y minería: la empresa norteamericana de Real del Monte y Pachuca. Tesis, Universidad Autónoma de Hidalgo, Hidalgo, México, p. 42.

20. Hemerobiblioteca del Mineral del Real del Monte. Fondo Norteamericano. Sección Serie Archivo Especial de la Dirección General, Subserie General, expediente 37. vol. 48.

21. González F, Grothe A, Salazar L. La industria minera de México. Volumen I. México: Imprenta y Fototipia de la Secretaría de Fomento; 1911. p. $112-115$.

22. Oviedo-Gámez B. Centro Cultural Nicolás Zavala, Museo de Medicina Laboral [Catálogo]. México: Archivo Histórico y Museo de Minería; 2005. p. 12. 